\title{
Optimization of Hot Free Forging Condition for the Uniformity of Forged Shape by Three Dimensional Rigid-plastic Finite Element Analysis
}

\author{
Kenji TAMURA and Jumpei TAJIMA ${ }^{11}$ \\ Corporate Research \& Development Laboratories, Sumitomo Metal Industries Ltd., Fuso-cho, Amagasaki 660-0891 Japan. \\ 1) Amagasaki Steel Works, Sumitomo Metal Industries Ltd., Higashimukoujima-nishino-cho, Amagasaki 660-0856 Japan.
}

(Received on October 13, 2000; accepted in final form on December 8, 2000)

\begin{abstract}
Focusing on the rough forging stage of the hot free forging process, the influence of the operational conditions on the occurrence of concave defects was first numerically analyzed using a three dimensional rigidplastic finite element method. The validity of the analysis was examined by laboratory experiments using lead billets and the analytical results are in good agreement with experimental results. As a result, the mechanism through which the concave defects appear was clarified, and it was revealed that by applying only a small curvature to a plane surface of an anvil, the concave defects is enormously improved. Moreover, it was found that the new anvil geometry makes it possible to increase the productivity.
\end{abstract}

KEY WORDS: free forging; finite element method; plastic deformation; anvil design.

\section{Introduction}

In general, it is difficult to use a cast ingot directly to make products because of its poor strength and toughness. In order to improve the toughness and ductility, the cast ingot is often subjected to hot rough-forming by which the coarse grain of the cast structure is refined. After this process, the toughness and ductility will meet the required values.

There are two methods of rough-forming; rolling and free forging. By one of these methods, an ingot is deformed into a long intermediate billet which has a uniform cross section geometry in the longitudinal direction for the preparation of the final forming. Among the two rough-forming methods, the free forging method requires less initial investment and tool cost than the rolling method due to its simplicity, and thus it is more likely to be adopted on the production line. It also requires less number of changes of operational stages.

The free forging process for rough-forming is roughly divided into two stages; rough forging and spiral forging. The former does most of the reduction of the workpiece. Although the final dimensional precision depends upon the latter stage, it is very important to form the billets in a good dimensional precision by the rough forging stage in order to prevent forging defects from occurring and also to ensure the dimensional precision in the final forging stage.

One of the most fatal deformations in the rough forging stage is a localized concave deformation which arises on a forged surface. Although it is known by experience that a concave defect somehow disappears by reducing productivity, i.e. by reducing feed and forging stroke in the operation, it is often the case in the production line that productivity has a priority to the dimensional precision and local concaves often remain on the forged surface. If the depth of the concave defect is large, it is necessary to grind the rough forged billets before the final forging stage, which leads to an increase in the production cost.

In the free forging process, the workpiece is intermittently forged by using an anvil, and a kind of deformation discontinuity inevitably arises in between the deformed zones by $N$-th and $(N+1)$-th shots, and it is assumed that this may be one of the causes of the concave defects. The geometry of the anvil generally used in free forging is flat, and this may accelerate the generation of concave defects because it is suspected that dead metal exists under the flat tool surface whereas deformation in the vicinity of both edges of the anvil is free.

Regarding free forging, there are some research works in which quantitative attention is focussed on such as microstructural evolution, ${ }^{1)}$ strain distribution, ${ }^{2-4)}$ or forging cracks. 5) Unfortunately, however, there is no quantitative study regarding the influences of operational conditions and tool geometry on the uniformity of deformation throughout rough forging, and thus, the operational conditions have been left to be determined by the operator's experience and intuition.

In this paper, the influence of the operational conditions on the occurrence of concave defects in the rough forging was first numerically analyzed using a three dimensional rigid-plastic finite element method. The validity of the analysis was then examined by laboratory experiments using lead billets. By analyzing the numerical results, the mechanism through which the concave defects appear was 


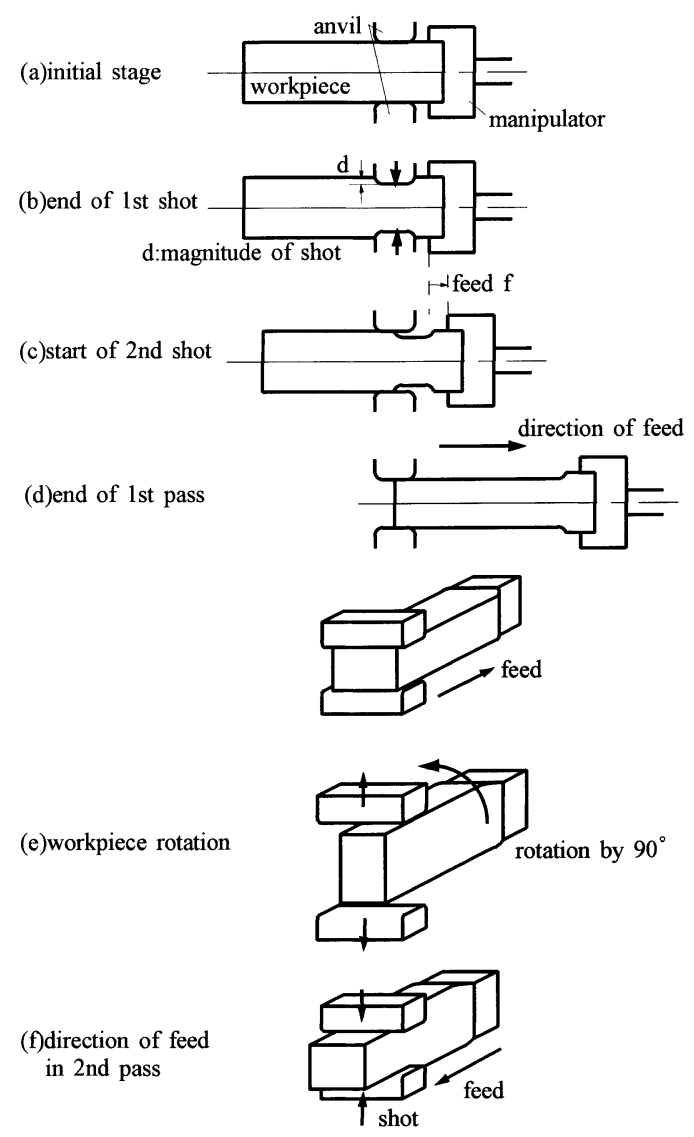

Fig. 1. Schematic drawing of rough-forging.

clarified, and the anvil geometry was optimized for suppressing the occurrence of concave defects. The new anvil geometry allows for a small curvature on the surface. Numerical analysis on the optimized anvil has made it quantitatively clear that the dead metal disappears and the finished geometry of the forged surface is greatly improved. It was also found that new anvil geometry makes it possible to increase productivity. The details are reported in the following sections.

\section{Numerical Analysis}

The conditions for the numerical analysis for the simulation of the concave defects in the rough forging with a flat anvil generally used on the production line are as follows: As shown in Fig. 1, a workpiece held with a manipulator is subjected to forging, where $d$ denotes the magnitude of shot and $f$ the feed per shot. The forging is repeated until the final shot on either end of the workpiece is completed. Then, the workpiece is usually rotated by 90 degrees and is subjected to a 2 nd pass, forging the side surface of the workpiece in the 1st pass, and the same applies to the following passes. In the numerical analysis in our paper, attention was focused on the 1st pass on the assumption that essential deformations during the passes keep repeating the same deformations as in the 1 st pass. Table 1 shows the conditions for the analysis and examples of the mesh. The software used for the analysis is a code for three dimensional rigid-plastic finite element analysis developed on our own $^{6,7)}$ based on a two dimensional code "RIPLS-FORGE" developed by Osakada et al. ${ }^{8)} \mathrm{A}$ quarter model was adopted
Table 1. Analytical conditions for analysis and examples of meshes for material and anvil.

\begin{tabular}{|l|l|}
\hline Thermal condition for material & Uniform temperature at $1240{ }^{\circ} \mathrm{C}$ \\
\hline Flow stress (stainless steel) & $\bar{\sigma}=43.9 \times \bar{\varepsilon}^{0.24} \times \bar{\varepsilon}^{0.0}(\mathrm{MPa})$ \\
\hline Initial dimensions of a workpiece & $500 \mathrm{H} \times 500 \mathrm{~W} \times 2000 \mathrm{~L}(\mathrm{~mm})$ \\
\hline Dimensions of a flat anvil & Width $=360 \mathrm{~mm}$, Flat part length $=180 \mathrm{~mm}$ \\
\hline Coefficient of friction & 0.2 \\
\hline Press speed & $5.0 \mathrm{~mm} / \mathrm{s}$ \\
\hline Feed f for each shot & $50,75,100,150,180 \mathrm{~mm}$ \\
\hline Magnitude of shot d for an anvil & $15,30,50 \mathrm{~mm}$ \\
\hline $\begin{array}{l}4800 \text { nodes } \\
3626 \text { elements }\end{array}$ \\
$\begin{array}{l}\text { An example of mesh for a workpiece. } \\
\text { (quarter model) }\end{array}$ \\
\hline
\end{tabular}

as the workpiece because of its symmetry and triangle patches were adopted to express the tool surface. In order to minimize a computational time for each case, mesh density in the longitudinal direction was carefully chosen and the mesh shown in the table, of which minimum element length in the longitudinal direction was approximately $12 \mathrm{~mm}$, was sufficient to predict the forged shape in good precision.

As attention was focused on the initial pass and also as the rough forging is generally finished in a short time, temperature drop of the billet was assumed to be nearly negligible. Therefore, uniform temperature of the billet was assumed throughout the analysis. Also here, strain rate hardening of the material was assumed to be almost negligible at the high temperature such as $1240^{\circ} \mathrm{C}$ and the strain-rate sensitivity of the material was approximated 0.0.

The ranges of two major operational parameters, i.e. feed $f$ and magnitude of shot $d$, are shown in Table 1, which are selected to cover real operational conditions. The lower bounds were determined by referring to the experimentally known values below which productivity drops sharply. The upper bounds were chosen by observing the initiation of the "tucking up" phenomenon of metal flow. If the values of two parameters exceed these upper bounds the metal flows over the anvil edge, which leads to forging defects. If the magnitude of shot $d$ exceeds the upper bound, the forging load becomes too large.

\section{Results}

Figure 2 shows an example of analytical result of a deformed shape, and it clearly shows that the initiation of localized concave deformation appears. Here, it is observed that the localized concaves appear at an interval that coincides with feed $f$. The reason for this cycle can be explained as follows: The surface of a workpiece portion, which was forged in the vicinity of the edge of the anvil in the previous shot, is brought down by metal flow in the next shot and remains as a concave. Then the same phenomenon arises in every shot and thus the localized concaves appear at the interval. Here, the mean value of the measure $h$ of each depth of these localized concaves was adopted to evaluate the effect of the following optimization.

As shown in Fig. 3(a), it was clarified that the smaller the feed is, the smaller the depth of localized concave is. 
Regarding the magnitude of shot $d$, as shown in Fig. 3(b), the smaller the value is the smaller the depth of localized concave is.

As a result, in order to improve the discontinuity of the deformation in rough forging with a pair of flat anvils, namely to reduce the depth of localized concave, it is necessary to select a small feed and a small magnitude of shot. However, to reduce the feed and the magnitude of shot means the decrease of productivity, which leads to an increase in the production cost. Therefore, in order to suppress the occurrence of concave defects without productivity drop, it is necessary to optimize not only the operational parameters but also the anvil geometry.

\section{Experimental Verification}

Experiments in our laboratory were carried out in order to verify the accuracy of the above numerical results, and the deformed workpiece shape and the influence of each operational parameter were compared.

Table 2 shows the experimental conditions and a photograph of the experimental facilities. A horizontal press, the maximum capacity of which is 100 ton, was used and pure lead was adopted as the material. All experiments were carried out in $1 / 5$ scale of real forging. In the following comparison shown in Fig. 4, the values of the experimental re-

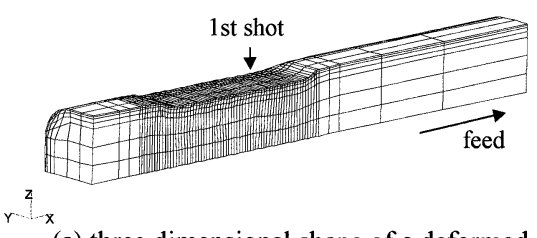

(a) three dimensional shape of a deformed mesh

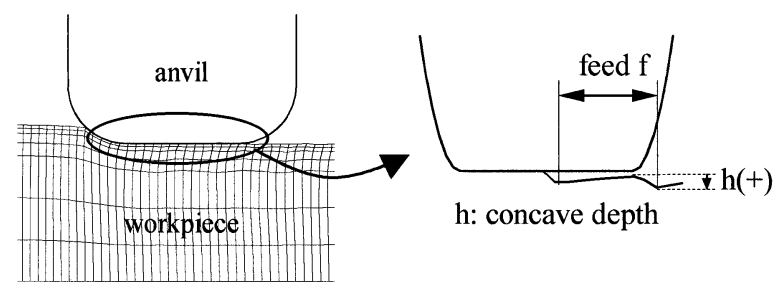

(b) configuration of workpiece and anvil at the end of 5th shot and definition of concave depth $\mathrm{h}$ (cross section at $\mathrm{y}=0$ )

Fig. 2. An example of a deformed mesh after 5th shot and localized concave deformation $(f=100 \mathrm{~mm}, d=30 \mathrm{~mm})$.

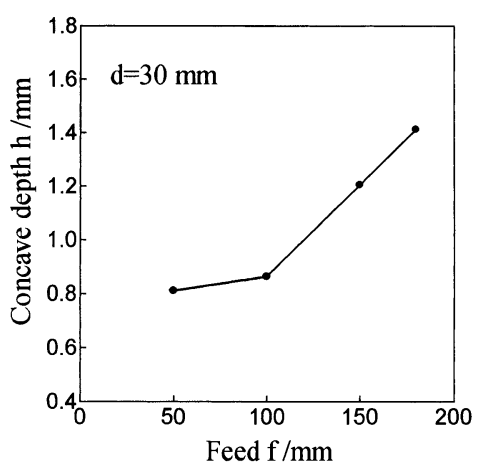

(a) sults are changed into $1 / 1$ scale of real forging and the measured forging loads of the experiments are changed by estimating the flow stress of pure lead to be $29.4 \mathrm{MPa}$.

Both in Figs. 4(a) and 4(b), the predicted deformation by the analysis was in good agreement with the experimental results, and thus, the analysis predicts the deformation in the rough forging well. Regarding the influence of each operational parameter, shown in Figs. 4(c) and 4(d), good agreement was also obtained between the numerical and experimental results. Moreover, as shown in Figs. 4(e) and 4(f), the predicted forging loads by the analysis were also in good agreement with the experimental results.

Here, it was reconfirmed that it is inevitable to reduce feed and reduction, namely productivity, to ensure uniformity of deformation in the forging using flat anvils.

Although the uneven side surface, shown in Fig. 4(b), is reformable as the surface is directly forged in the next pass, the forged surface can not be reformed. Therefore the forged surface deformation must be controlled while being forged. Also in this sense, it is very important to ensure uniformity of deformation of the forged surface.

As a result, it was quantitatively confirmed that it is sufficient not only to establish the fundamental scheme to improve the uniformity of deformation but also to estimate forging load characteristic in the rough forging by numerical analysis. In the following section, an optimization of the anvil geometry is carried out by analysis.

Table 2. Experimental conditions and the experimental facilities.

\begin{tabular}{|l|l|}
\hline Scale of experiment & $1 / 5$ scale of the real forging \\
\hline Material & Pure lead \\
\hline Initial dimensions of a workpiece & $100 \mathrm{H} \times 100 \mathrm{~W} \times 400 \mathrm{~L}(\mathrm{~mm})$ \\
\hline Dimensions of a flat anvil & Width $=72 \mathrm{~mm}$, Flat part length $=36 \mathrm{~mm}$ \\
\hline Feed f for each shot & $10,20,30 \mathrm{~mm}$ \\
\hline Magnitude of shot d for an anvil & $3,6,10 \mathrm{~mm}$ \\
\hline
\end{tabular}

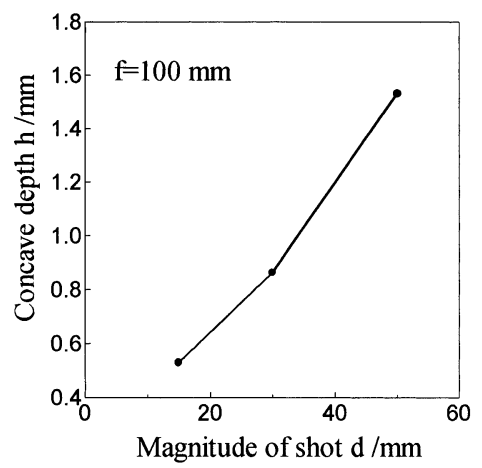

(b)

Fig. 3. Influences of operational parameters, (a) feed and (b) magnitude of shot, on concave depth. 


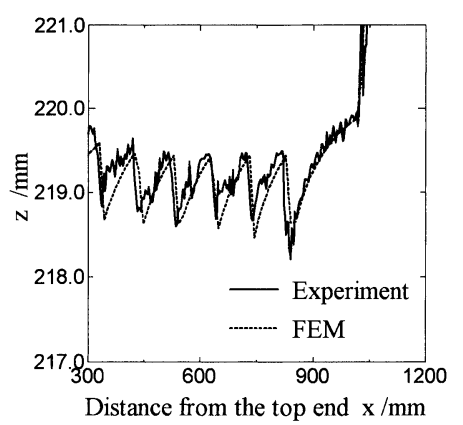

(a) deformation of the forged surface $(\mathrm{f}=100 \mathrm{~mm}, \mathrm{~d}=30 \mathrm{~mm})$

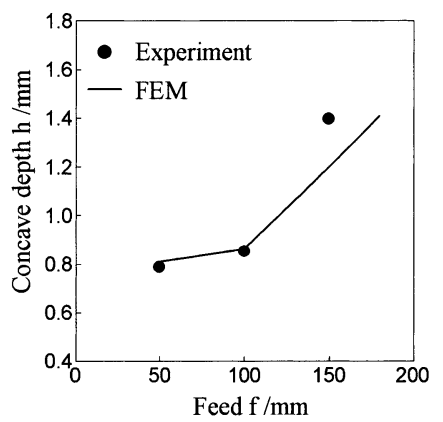

(c) influence of feed on concave depth $(\mathrm{d}=30 \mathrm{~mm})$

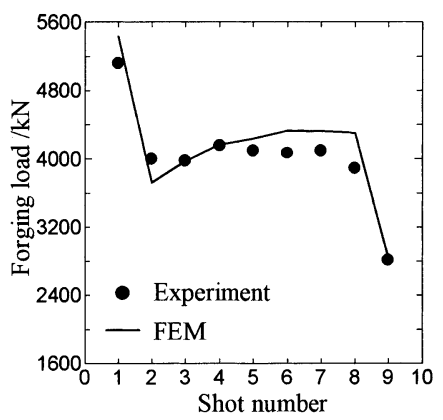

(e) forging loads by $\mathrm{f}=100 \mathrm{~mm}$ and $\mathrm{d}=30 \mathrm{~mm}$

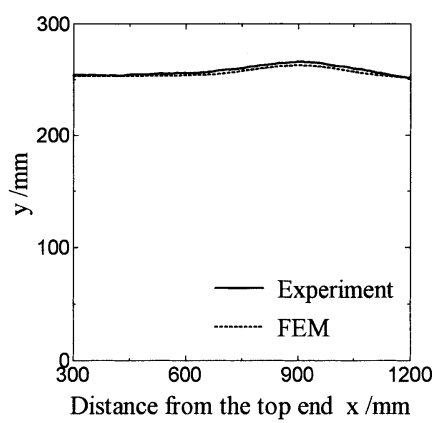

(b) deformation of the side surface $(\mathrm{f}=100 \mathrm{~mm}, \mathrm{~d}=30 \mathrm{~mm})$

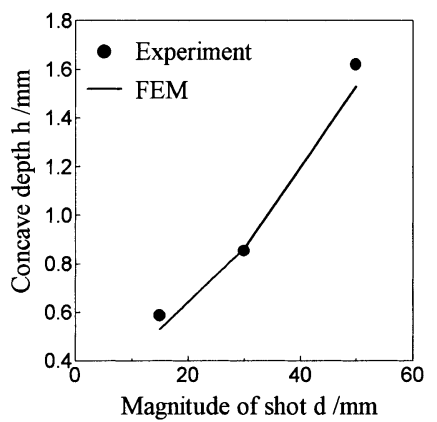

(d) influence of magnitude of shot on concave depth $(\mathrm{d}=30 \mathrm{~mm})$

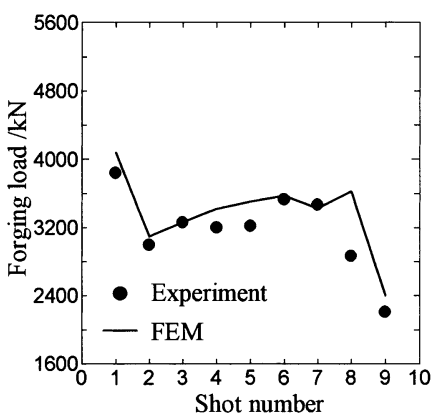

(f) forging loads by $\mathrm{f}=100 \mathrm{~mm}$ and $\mathrm{d}=15 \mathrm{~mm}$

Fig. 4. Comparison between analytical and experimental results.

\section{Optimization of Anvil Geometry by Numerical Analysis}

The mechanism through which the concave arises can be explained by Fig. 5. At the beginning of a shot, as shown in Fig. 5(a), the non-forged surface of the workpiece makes contact first with the flat tool surface. Then, the workpiece portion, which was forged with the curved edge of the anvil in the previous shot, gradually makes contact with the flat tool surface. Here as shown in Fig. 5(b), it was found that, in the workpiece portion which makes contact with the flat tool surface, dead metal exists where no plastic strain is obtained. As the dead metal region is pushed down as a rigid body with the anvil, the boundary portion between the previous and the present shot is brought down and finally the concave arises.

Therefore, in order to suppress the occurrence of concave defects, the authors have tried to reduce the dead metal by adopting a curved surface instead of a plane surface in relationship to the anvil. In the new anvil design, shown in Table 3, the plane surface of a conventional flat anvil was replaced with a curved surface, and the curvature of the surface $C s$ was varied in the range in the table. In evaluating the effect of the anvil design, the depth of concave was adopted. Here, the feed $\mathrm{f}$ was fixed to $100 \mathrm{~mm}$ and the magnitude of shot d was fixed to $30 \mathrm{~mm}$.

As shown in Fig. 6(a), the dead metal was considerably reduced with a curved surface anvil in comparison with the result of the flat anvil shown above in Fig. 5(b). Figure 6(b) shows examples of the predicted shape with different curvature of the anvil surface, including flat anvils. It is clearly shown that by adopting a curved surface, concave defects were greatly reduced and an almost uniform deformation was obtained with the anvil which had a curvature of $2.0 \times 10^{-4}$.

Therefore, it was found that by applying only a small curvature to the anvil surface, the dead metal is reduced and the continuity of deformation is enormously improved. This is a remarkable result though it is absolutely contrary to the conventional belief on the production line that flat anvils should be adopted to obtain a smooth deformed surface.

On the other hand, in the case that of a curvature 


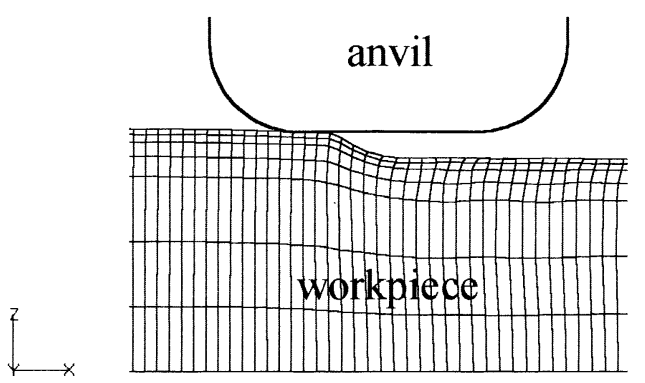

(a) configuration of anvil and workpiece at the start of 5 th shot

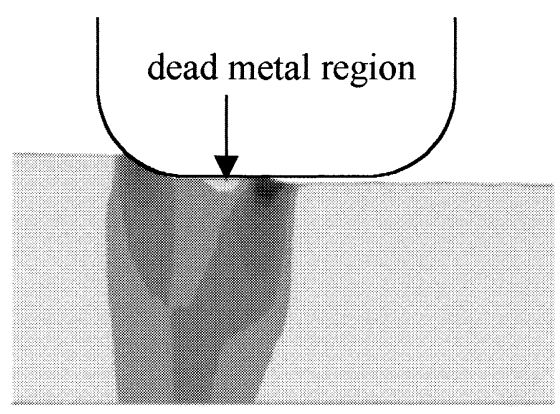

(b) distribution of equivalent strain rate in the middle stage of 5 th shot

Fig. 5. Initiation of localized concave deformation (cross section at $y=0)$.
$C s=8.0 \times 10^{-4}$, corrugation was found at the forged surface instead of concave. This means that if the curvature becomes too large, there are portions at the boundary of shots where the reduction is insufficient and the intensity of the corrugated surface becomes larger. Here, the depth of corrugation is expressed as a minus value of the depth to the concave area.

In summary, Fig. 6(c) shows the effect of the curvature on the depth of concave $h$. Here, the concave depth using a flat anvil is also shown. It is clear that even with the curvature $1.25 \times 10^{-4}$, which is the minimum value in this analysis, the depth $h$ decreases to a half the value of the concave depth using a flat anvil. And then depth $h$ reaches its minimum with curvature $2.0 \times 10^{-4}$, although small corrugation still remains, as shown in Fig. 6(b). Above this curvature, the depth $h$ is reversed and increases its absolute value because of the lack of reduction at the boundary of shots, as mentioned in Fig. 6(b). As a result, by choosing the curva-

Table 3. Parameters for anvil design.

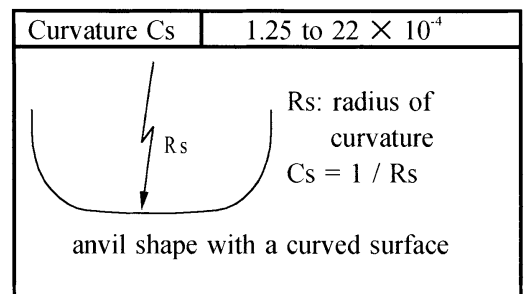

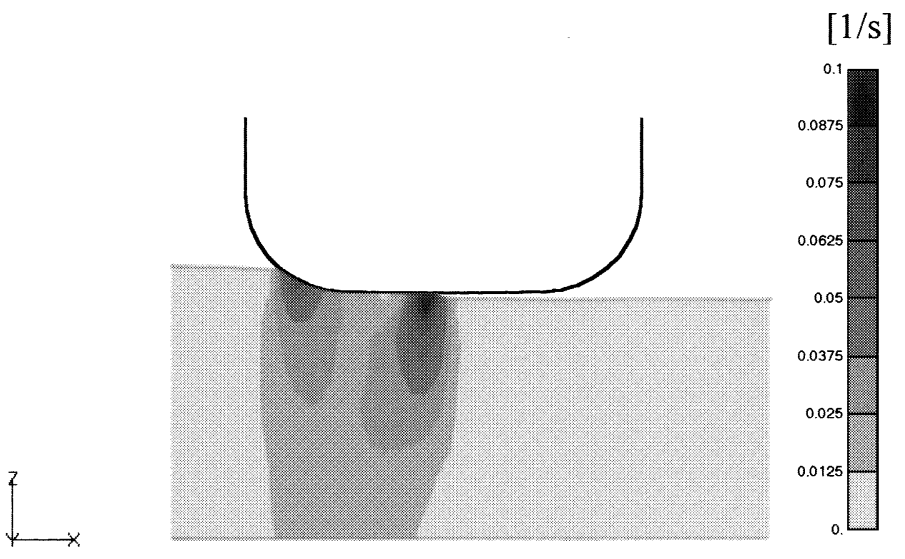

(a) distribution of equivalent strain rate

in the middle stage of 5 th shot $\left(\mathrm{Cs}=2.0 \times 10^{-4}\right)$

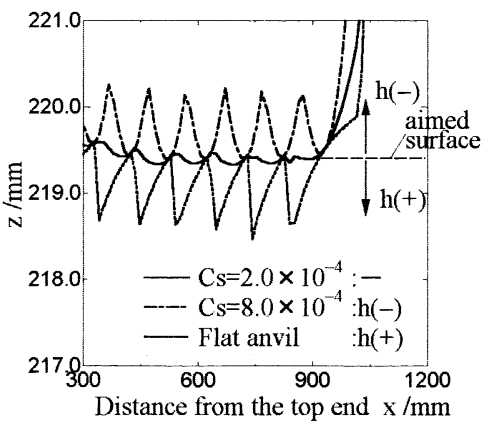

(b) deformation of the forged surface

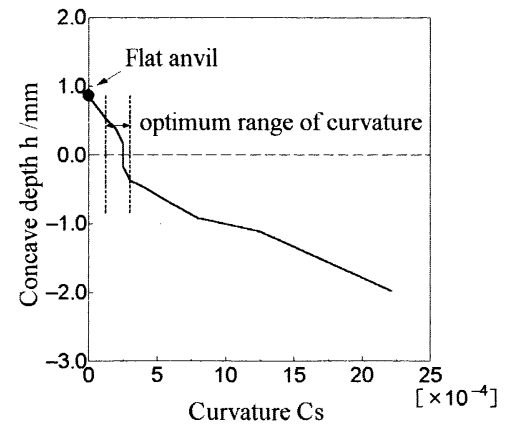

(c) influence of curvature on concave depth

Fig. 6. Effect of curved surface anvil. 
ture in the range shown in Fig. 6(c), the depth $h$ becomes no more than a half the value of the concave depth using a flat anvil.

Besides, as shown in Fig. 7, in the case that the curvature $C s$ is $2.0 \times 10^{-4}$, by which the concave depth $h$ is the minimum in Fig. 6(c), it was found that it is sufficiently applicable to take $180 \mathrm{~mm}$ as the feed which results in a smaller unevenness than the concave depth using flat anvils by $50 \mathrm{~mm}$ feed.

As a result, it was quantitatively clarified that by adopting the newly designed anvil, it is possible not only to ensure the uniformity of the deformation but also to employ a

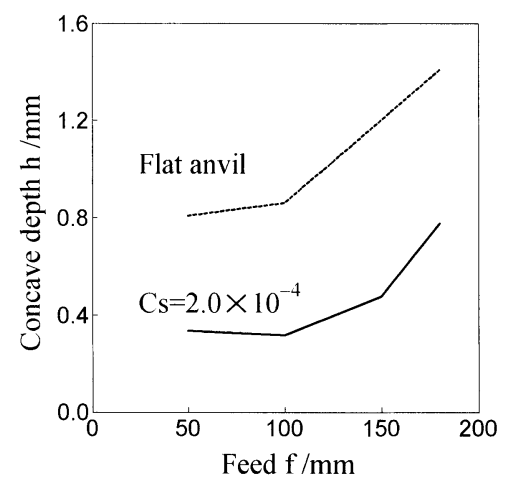

Fig. 7. Comparison of the influence of feed on concave depth between forgings with flat anvils and curved surface anvils $(d=30 \mathrm{~mm})$.

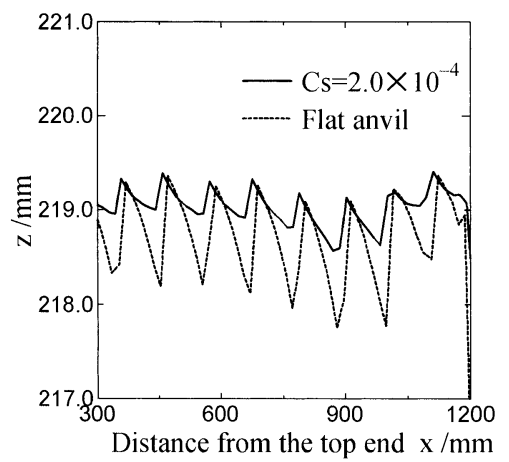

(a) large feed, which results in high productivity.

\section{Discussion}

The previous analyses were carried out regarding the $1 \mathrm{st}$ pass of rough forging. Here, as mentioned in sec. 2, the authors assumed that the essential deformations for the passes repeat the deformation of the 1 st pass. In this section, the validity of the assumption is examined by numerical analysis in the following figure.

Figure 8 shows the analytical results of the surface after rough forging up to the final pass, i.e. the 6th pass both with anvils equipped with a curved surface of curvature $C s$ is $2.0 \times 10^{-4}$ and using flat anvils. Obviously, by adopting the curved surface anvils, it is possible to complete the rough forging stage with small depth $h$ at the forged surface. Therefore, it can be concluded that the essential deformations in the later passes repeat the deformation in the $1 \mathrm{st}$ pass and thus, it is sufficient to focus attention on the 1 st pass to ensure the uniformity of the deformation.

Secondly, the loading characteristic of the newly designed anvil was examined by numerical analysis. Regarding productivity, forging load is one of the most important factors. If the load becomes too large, the reduction does not meet the aimed value because the forging load exceeds the press capacity, or the press motion becomes slow.

Figure 9(a) shows the forging load of the 1st shot which

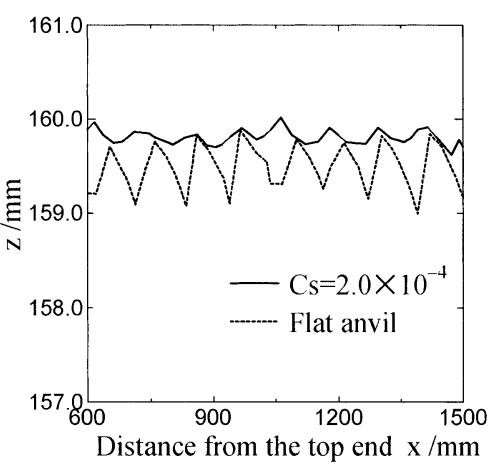

(b)

Fig. 8. Comparison of the forged surface deformation (a) in the 2 nd pass and (b) in the final pass between forgings with flat anvils and curved surface anvils.

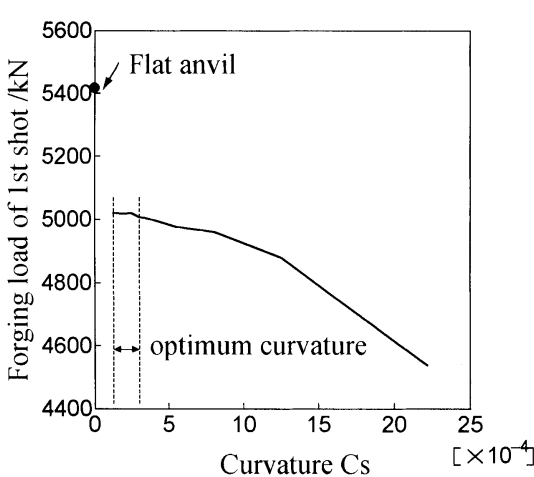

(a) relationship between curvature and forging load of 1 st shot

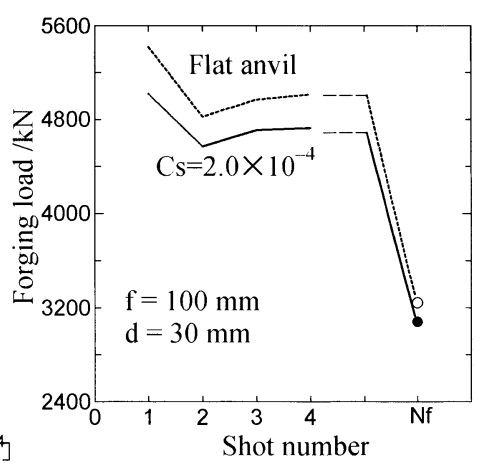

(b) comparison of forging loads throughout 1 st pass between forgings with flat anvils and curved surface anvils $\left(\mathrm{Cs}=2.0 \times 10^{-4}\right)$

Fig. 9. Effect of curved surface anvil on the forging load. 


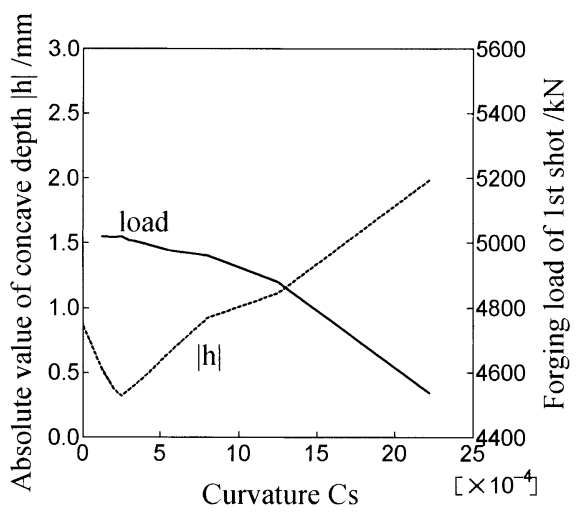

Fig. 10. Relationship between concave depth and forging load in forging with curved surface anvils.

is the largest in a pass because the workpiece is forged with the whole tool surface. It was revealed that the curved surface anvil has an effect to reduce the forging load of the 1 st shot and the larger the curvature of the curved surface is, the smaller the load becomes. Therefore, by choosing a large curvature of the curved surface within the range that the dimensional precision is specifically allowed in the previous section, the load characteristic is able to be improved at the same time with the improvement of the uniformity of the deformation.

Figure 9(b) shows the comparison of the forging loads in the $1 \mathrm{st}$ pass by $180 \mathrm{~mm}$ feed and $30 \mathrm{~mm}$ magnitude of shot between the forging with the curved surface anvils with $C s=2.0 \times 10^{-4}$ and the forging with flat anvils. It is clearly shown that the loads with the curved surface anvils are all smaller than those with the flat anvils throughout the pass. This means that by adopting the newly designed anvils to the production line, the forging can be completed quickly with fast press motion, which results in high productivity. Here, Nf means the shot number of the last shot in the 1st pass and it is clearly shown that the forging load drops when forging reaches at the free end of the workpiece.

As a result, Fig. 10 shows the relationship between the absolute value of the depth of the concave area and the forging load of the 1 st shot corresponding to the curvature of a curved surface anvil. It is a clever choice at the plant to determine the anvil design which satisfies both the uniformity of the deformation and the forging load.

In summary, it was quantitatively revealed that the curved surface not only enables the production line to apply a large feed with ensuring the uniformity of the deformation of the forged surface, but it also is excellent in the load characteristic. As a result, it is possible with the new anvils to realize both the uniform deformation and high productivity with minimum energy consumption. The discussion on the forging load is finished as it is not the main subject of this paper.

\section{Conclusions}

Focusing on the rough forging stage of the hot free forging process, the influence of the operational conditions on the occurrence of concave defects and the optimization of the anvil geometry were examined by numerical analysis with a three-dimensional finite element method. As a result, it was revealed that by applying only a small curvature to a plane surface of an anvil, the discontinuity of the deformation, i.e., the concave defects is enormously improved and the optimum range of the curvature was quantitatively specified. Moreover, it was found that the new anvil geometry makes it possible to increase the productivity. The main points are the followings:

(1) A method of the numerical analysis for examination of the deformation in hot free forging was established. The analytical results quantitatively agree with the experimental results and it is sufficient to optimize the operational conditions and anvil design by this analysis.

(2) It was confirmed that the essential deformations for the passes repeat the deformation of the 1st pass, and then it is possible to examine the optimization of the whole process by evaluating only the 1 st pass.

(3) In order to reduce the depth of localized concave area in rough forging using a pair of flat anvils, it is necessary to select a small magnitude of feed and a small magnitude of shot. However, to reduce the feed and the magnitude of shot means a decrease in productivity which leads to an increase in the production cost. Therefore, in order to suppress the occurrence of concave defects without a productivity drop, it is necessary to optimize not only the operational parameters but also the anvil geometry.

(4) In order to ensure the uniformity of the deformation, it is very important to reduce the dead metal and it was revealed that by applying only a small curvature to the plane surface of an anvil, the discontinuity of the deformation, i.e., the concave defect is enormously improved. The optimum range of the curvature was quantitatively specified.

(5) It was found that the curved surface anvil is excellent not only in the uniformity of the deformation, but also in the load characteristic. However, in optimizing the forging load characteristic, other discussions are necessary to focus on this subject.

(6) In summary, the fundamental scheme to optimize pass schedule and tool design for the uniformity of the deformation in the rough forging was clarified.

\section{Acknowledgements}

The authors would like to acknowledge the advice by Dr. M. Akiyama in constructing and writing this paper.

\section{REFERENCES}

1) C. A. Dandre, S. M. Roberts, R. W. Evans and R. C. Reed: Mater. Sci. Technol., 16 (2000), 14.

2) B. V. Kiefer and K. N. Shah: Trans. ASME. J. Eng. Mater. Tech., 112 (1990), 477.

3) C. Hung and S. Kobayashi: Trans. ASME. J. Eng. Ind., 114 (1992), 459.

4) R. Paukert: Annals of the CIRP, 32 (1983), 211.

5) K. Tamura, Y. Hida, T. Fukuyasu and M. Yamada: Proc. of Plasticity '95, ed. by S. Tanimura et al., Gordon and Breach Publishers, Luxembourg, (1995), 907.

6) K. Tamura and J. Tajima: Proc. of Plasticity '97, ed. by A. S. Khan, NEAT PRESS, Maryland, (1997), 411.

7) K. Yamamoto: Proc. of 13th Int. Forgemasters Meeting, Korea Heavy Industries \& Construction Co., Ltd. et al., Shogen, (1997), 115 .

8) K. Osakada, J. Nakano and K. Mori: Int. J. Mech. Sci., 24 (1982), 459. 